\title{
Tangence
}

\section{Vitesse et inertie : les deux univers de L'île de béton}

\section{Christine Legault}

Numéro 55, septembre 1997

La vitesse

URI : https://id.erudit.org/iderudit/025944ar

DOI : https://doi.org/10.7202/025944ar

Aller au sommaire du numéro

Éditeur(s)

Tangence

ISSN

0226-9554 (imprimé)

1710-0305 (numérique)

Découvrir la revue

Citer cet article

Legault, C. (1997). Vitesse et inertie : les deux univers de L'île de béton.

Tangence, (55), 8-17. https://doi.org/10.7202/025944ar d'utilisation que vous pouvez consulter en ligne.

https://apropos.erudit.org/fr/usagers/politique-dutilisation/ 


\section{Vitesse et inertie: les deux univers de L'île de béton \\ Christine Legault}

Qu'attendrons-nous lorsque nous n'aurons plus besoin d'attendre pour arriver?

Paul Virilio

À l'heure actuelle, le T.G.V. prend deux heures pour atteindre Lyon en provenance de Paris. En 1832, il fallait compter trois jours et demi pour franchir cette même distance en diligence ${ }^{1}$. Alors qu'avant 1866 , année de la première communication télégraphique transatlantique, un message d'outre-mer prenait plusieurs semaines pour parvenir à destination, la transmission des messages se fait maintenant instantanément, à 300000 kilomètres par seconde. Les délais sont abolis et il devient même possible de se réunir à distance dans le cyberespace. Depuis environ un siècle et demi, les innovations technologiques - du train à l'ordinateur en passant entre autres par l'automobile, le téléphone, l'avion et la vidéo - ont engendré une accélération des transports et des communications. Auparavant statique, l'univers, constamment métamorphosé par l'accroissement de la vitesse, est devenu mobile. La prolifération des machines et des infrastructures technologiques ainsi que la rapidité des déplacements et les impressions cinétiques que ceux-ci suscitent transforment l'environnement, brouillent les repères qui le jalonnent. Les distances se rapprochent sous l'effet de la vitesse et, par le fait même, "'horizon ne cesse de fuir au loin "2. L'accélération des moyens de transport et de communication remet en outre en question le concept de temps. Elle a conduit par exemple à l'invention de nouvelles divisions temporelles, "microseconde en 1950, nanoseconde en 1965 (milliardième de seconde), femto-seconde en 1990

1 Claude Pichois, Vitesse et vision du monde, Neuchâtel, Éditions de la Baconnière, 1973, p. 21.

2 Paul Virilio, L'borizon négatif: essai de dromoscopie, Paris, Éditions Galilée, 1984 , p. 156. 
(millionième de seconde) [sic] et bientôt l'attoseconde vers 2020 (milliardième de milliardième de seconde)" 3 . En fait, l'accélération de la vitesse n'entraîne pas seulement une restructuration de l'espace et du temps, mais affecte le rapport de l'être au monde, les relations sociales et la vie quotidienne. Toutes les sphères de l'activité humaine ont été marquées par la vitesse au cours des dernières décennies. Par exemple, les sports de vitesse se sont substitués aux sports de force, les modes d'expression artistique - avec entre autres le pop art, l'infographie et le vidéoclip - se sont diversifiés et les stratégies militaires et politiques ont dû être entièrement repensées en raison de la nouvelle intempérance médiatique. On peut ainsi affirmer, avec Beaud et de Loisy, que la vitesse façonne le monde, qu'elle s'inscrit depuis le début du siècle comme "vecteur de mutation des connaissances et des attitudes" 4 .

Issu d'un contexte socioculturel dont il ne peut être dissocié, le texte littéraire porte aussi l'empreinte de cette accélération contemporaine. En plus des divers récits et poèmes qui exaltent ou condamnent la vitesse et les machines qui la permettent - on peut en trouver des exemples chez des auteurs aussi célèbres que Nerval, Verlaine, Hugo, Du Camp, Dickinson et Whitman nombre d'écrivains rendent compte des impressions cinétiques, de la restructuration du temps et de l'espace ainsi que des transformations socioculturelles engendrées par l'accélération des moyens de transport et de communication ${ }^{5}$. L'intérêt du texte sur lequel je m'attarderai, L'île de béton de James Graham Ballard ${ }^{6}$, vient de ce qu'il explore au contraire un retour à un apparent statisme dans un monde en constant mouvement. Immobilisé et isolé malgré lui, le héros de ce roman remet en effet en question un mode de vie et un univers réglés par la vitesse.

L'île de béton débute par un accident. Robert Maitland, un architecte âgé de 35 ans, roule en Jaguar sur l'autoroute à plus de 110 kilomètres-heure et perd le contrôle de son automobile

3 Marie-Claude Beaud et Jean de Loisy, "Une mise en scène", avant-propos de La vitesse, ouvrage collectif de la Fondation Cartier, Paris, Flammarion, 1991, p. 5.

4 Ibid.

5 Claude Pichois, op. cit., p. 25.

6 J. G. Ballard, Lî̀le de béton, trad. de l'anglais par Georges Fradier, Paris, Christian Bourgois Éditeur, 1974, 186 p. Les citations suivies de numéros de pages entre parenthèses proviennent toutes de cette édition. 
lorsqu'un pneu éclate. La voiture s'immobilise sur un terrain abandonné long de deux cents mètres et ceinturé de trois routes convergentes. Malgré ses efforts persistants pour s'évader de cet îlot jonché d'ordures, de mauvaises herbes et de ruines, Maitland y reste confiné jusqu'à la fin du récit, c'est-à-dire pendant plus d'une semaine. Comme Robinson Crusoé sur son île, il explore son nouvel environnement, trouve à boire, à manger et à se loger, combat la douleur, la fièvre et l'épuisement et fait des rencontres: celles de Proctor le clochard et de Jane la fille des rues, des êtres marginalisés, socialement inadaptables. Deux univers s'opposent dans le roman: celui des autoroutes et donc des trajets, de la vitesse, de l'automation, du désordre et de l'entropie - monde fugitif, quasi indescriptible pour le héros - et celui de cette île recouverte non pas de béton mais d'herbe, système presque clos, figé dans un statisme révolu. Exilé contre son gré tout en soupçonnant qu'il a luimême souhaité et provoqué cette situation, Maitland n'a d'autre choix que de se familiariser avec les lieux, avec cette île "vraiment étrangère au réseau routier, infiniment plus ancienne que toute la région" (p. 69). Miquel affirme que

par une mystérieuse application du principe d'entropie, tout monde tend, une fois formé, à se couper de la vie réelle, en s'en dédoublant et voulant se maintenir sous une forme immuable échappant au changement : il se transforme insensiblement en un "autre monde " clos, éminemment conservateur. ${ }^{7}$

Troublé à l'idée de retrouver sa femme si tôt après avoir quitté sa maîtresse, Maitland échoue sur cette île "réellement coupée du monde extérieur" (p. 13). Espèce de machine à remonter le temps, la voiture le fait retourner dans le passé, "pas seulement dans le passé de l'île, mais surtout dans le sien" (p. 70). Alors qu'il explore le terrain en ruine, l'architecte se rappelle en effet son enfance et certaines expériences passées, remet en question sa façon de vivre, ses relations amoureuses, familiales et amicales, son travail. Ses échecs répétés pour quitter l'île traduisent une ambivalence quant à son véritable désir de regagner une vie active. Vers la fin du roman, il affirme même ne plus vouloir partir: "Non, je ne veux pas! Je veux rester. En tout cas, pour le moment, je veux rester ici" (p. 169). En fait, l'île s'avère peut-être le seul endroit pouvant donner à Maitland le temps de penser, de

7 Christian Miquel, Critique de la modernité: l'exil et le social, Paris, Éditions l'Harmattan, coll. "Logiques sociales ", 1992, p. 64-65. 
s'arrêter et de faire le point. Fuyant, toujours tourné vers le futur, l'univers des autoroutes, où tout se décide en quelques microsecondes, comme l'accident du héros, enlève aux individus tout pouvoir décisionnel. Dans son nouvel environnement, Maitland acquiert au contraire un certain contrôle. C'est du moins son principal objectif : "cette volonté de survivre, de mâ̂triser l'île et d'en exploiter les maigres ressources, c'était un but plus important que de s'échapper" (p. 65).

Habitué à la vitesse, le héros de L'ile de béton doit adapter ses perceptions à sa nouvelle situation. L'inertie qui lui est imposée bouleverse sa conception du temps et de l'espace. Tandis qu'il pouvait auparavant parcourir sans effort plusieurs kilomètres en voiture, il lui est maintenant difficile de traverser l'île à pied. Les 15 kilomètres qui le séparent de sa famille lui paraissent infranchissables, à un tel point qu'il commence à oublier ses proches devenus trop lointains: "Il lui fallait faire un effort pour penser à sa femme, à son fils, à Helen et pour retrouver leurs traits... des visages qui s'éloignaient, qui se perdaient dans la grisaille comme les nuages au-dessus de White City" (p. 150). Alors qu'une accélération des transports rapproche les distances, la perte de vitesse dans ce roman a pour effet d'accroître leur ampleur. Conséquemment, l'horizon de Maitland se rétrécit. Après quelques jours sur son île, il a en effet l'impression que celle-ci devient plus vaste et que le réseau routier s'éloigne: "Maitland ne se lassait pas de regarder rouler les voitures, en haut des remblais qui lui semblaient plus lointains aujourd'hui: ils reculaient lentement de tous les côtés. En revanche, l'île paraissait plus grande, sa végétation plus dense, plus belle" (p. 122). Graduellement réduite depuis l'invention du train par l'accélération des transports, la distance redevient dans L'île de béton un paramètre déterminant, un obstacle.

Depuis les minutes qui ont suivi son accident et subséquemment pendant plusieurs jours, même après le vol de sa montre par Proctor, Maitland regarde ou évalue à maintes reprises l'heure et la date. Notant d'abord le déroulement du temps à la minute près, il devient de moins en moins précis pour finalement constater qu'il ignore depuis combien de jours il se trouve sur l'île: "Quel jour était-ce? Mercredi, vendredi peut-être" (p. 163). Après la disparition de sa montre, il estime l'heure en se fiant à la densité du trafic routier ou à des facteurs environnementaux cycliques comme la position du soleil, la température, la brume, la rosée et 
la luminosité du ciel. Le temps semble donc s'écouler selon deux vecteurs opposés: l'un rapide, dirigé vers un futur insaisissable et lautre plus lent, favorisant la réminiscence. Confondus par ces deux perceptions inverses, Maitland perd la notion du temps. Son nouveau mode de vie au ralenti brouille en fait tous ses repères spatio-temporels. Peu après son accident, il tente par exemple de traverser la chaussée, évalue mal la vitesse des voitures et la distance qui les sépare et se fait blesser. Un peu plus tard, il ne réagit pas assez vite aux signes encourageants d'un conducteur qui aurait pu lui venir en aide: "quand Maitland comprit, quant il commença à remuer, l'Américain avait déjà accéléré en saluant poliment" (p. 35). Si Maitland échoue dans ses tentatives de quitter l'île et est ignoré des conducteurs, c'est avant tout parce que la différence de vitesse entre les deux univers est trop importante. Bien que contigus, ceux-ci restent, sauf accidentellement, isolés l'un de l'autre. En effet, la vitesse des voitures et les infrastructures routières empêchent les conducteurs de s'arrêter:

D'ailleurs ici il leur serait pratiquement impossible de ralentir. Derrière, la pression était trop forte, les voitures venaient d'échapper aux longs bouchons qui bloquaient régulièrement l'échangeur aux heures de pointe. Pas question de s'arrêter, il fallait rouler. (p. 17)

En outre, "la vitesse doue le paysage de la mobilité dont est affecté le voyageur " ${ }^{8}$. Plus les voitures roulent vite, plus les impressions cinétiques se succèdent, donnant à l'environnement davantage de mobilité. Voyageant à grande vitesse, les conducteurs de Lîle de béton n'ont "d'yeux que pour les panneaux indicateurs et la jonction du périphérique» (p. 16) et ne sont donc pas en mesure de déchiffrer les messages que Maitland laisse en bordure de la route, bien que ceux-ci soient écrits en style télégraphique, et que Proctor, désireux de protéger son monde clos, se hâte d'effacer.

Les deux univers de L'v̂le de béton présentent des dangers pour la vie. Trop ordonné ou excessivement chaotique, l'un et l'autre peuvent entraîner la mort. Comme l'affirme Henri Atlan,

Une éthique vraie $[\ldots]$ serait la loi qui nous permettrait à chaque instant de savoir comment intervenir dans ce combat incessant entre la vie et la mort, l'ordre et le désordre, en sorte d'éviter

8. Claude Pichois, op. cit., p. 28. 
toujours un triomphe définitif de l'un quelconque sur l'autre, qui est, en fait, l'une des deux façons de mourir complètement, si l'on peut dire, par arrêt du processus soit dans un ordre définitivement établi, inamovible, soit dans un désordre total. ${ }^{9}$

La vie de Maitland est en premier lieu menacée par son "chromosome de brute" (p. 9) qui l'incite à conduire vite, à s'autodétruire dans un accident au cours duquel l'ordre routier est perturbé. En état de choc, il s'étonne de sortir indemne de cette expérience traumatisante. Proctor a moins de chance que lui. Machine d'un autre temps sur laquelle Maitland s'assoit comme sur le dos d'une bête afin de se déplacer avec plus d'aisance et de rapidité, Proctor est victime de la vitesse chaotique du réseau routier. Trop lent à réagir, son "cerveau paralysé" (p. 164) ne peut en effet rivaliser avec la célérité technologique. En plus des accidents qu'elles engendrent, les voitures remettent aussi en question le rôle de l'être humain dans son milieu. Leur multiplicité, leur omniprésence et l'importance accordée à la vitesse sur le réseau routier placent les personnages sous le contrôle de la technologie. Les êtres deviennent plus mécaniques, s'automatisent. C'est ainsi que Proctor sert de véhicule, que les passagers d'un car sont comparés à des "mannequins" (p. 12), que Jane, en vendant son corps, devient une femme-objet et que le visage de Maitland imite la froideur de l'automate: "Il fit la grimace pour essayer de donner une expression à cette face blafarde immobile. Plus une goutte de sang dans la lourde mâchoire, dans les joues dures" (p. 9). Alors que le corps perd ses caractéristiques humaines, ce sont les voitures qui se les approprient. Après le premier accident de Maitland, la description de la Jaguar évoque par exemple celle d'un corps à l'agonie: "Le radiateur écrasé éructait des jets de vapeur sifflante qui retombait en crachats d'eau rouillée. Du moteur sortait un rugissement caverneux, la mécanique vomissait son dernier râle” (p. 8). C'est d'ailleurs en quelque sorte la voiture qui redonne vie à Maitland puisque celui-ci se sent ranimé en buvant l'eau du radiateur. Par conséquent, bien qu'elle permette une accélération des déplacements et rende ainsi les individus plus fonctionnels, l'automobile manifeste sa supériorité en morcelant le corps, comme lors des accidents, ou en brouillant la frontière entre le mécanique et l'organique.

9 Henri Atlan, Entre le cristal et la fumée. Essai sur lorganisation du vivant, Paris, Seuil, coll. "Points", p. 58. 
14

L'univers statique de l'île ne semble pas davantage propice à la vie humaine. À peine blessé à la suite de son accident, Maitland se détériore peu à peu. La douleur l'obsède, ses blessures, maintes fois évoquées, s'aggravent: "Chaque jour qui passait lui enlevait une fraction de ses forces, et maintenant les herbes dansaient autour de lui avec des ricanements comme une foule hostile" (p. 125). Ce n'est plus dans ce cas-ci l'omniprésence de la technologie qui met en danger l'existence humaine, mais des matières organiques surabondantes, oppressantes. Cet environnement abritant un ancien cimetière évoque d'une part un retour dans un passé reculé, antérieur aux civilisations modernes. D'autre part, il annonce peut-être également un futur apocalyptique sur lequel je reviendrai plus loin. Qu'il se trouve dans l'un ou l'autre de ses deux pôles temporels, Maitland perd de sa vitalité. Soumis aux effets de l'inertie ambiante, son corps fonctionne au ralenti, ses organes deviennent inactifs: "Au-delà de la faim, audelà de l'épuisement, il était parvenu à l'état où les lois de la physiologie étaient suspendues, avec toutes les règles du métabolisme des besoins et des réactions" (p. 172). Cette paralysie affecte également les facultés mentales du héros. Elle lui permet de penser, mais il a "le cerveau vide" (p. 161), veut partir "à l'aventure en vagabondant tout seul dans la cité déserte de son esprit " (p. 145). C'est peut-être d'ailleurs en raison de son retard mental et d'un déficit visuel limitant ses horizons que Proctor se satisfait d'un mode de vie stagnant. Laissées à l'abandon, immobilisées, les voitures subissent le même sort que le corps de Maitland. Elles rouillent, se déglinguent, sont englouties sous la végétation, deviennent un tas de ferraille. Pour l'être humain comme pour la technologie, ce monde clos, inerte, conduit à la mort. Maitland en est du reste conscient et dit au clochard: "Proctor... tu attends ma mort, voilà ce que tu attends" (p. 166). Les deux processus systémiques pouvant provoquer la mort, tels que décrits par Henri Atlan, menacent donc les personnages du roman. Entre deux univers peu propices à la vie, ceux-ci paraissent en effet condamnés.

De la fin des années soixante jusqu'à la fin des années soixante-dix, Ballard a contribué, avec entre autres Michael 
Moorcock et Brian Aldiss, au magazine littéraire New Worlds. Les principaux écrivains qui ont collaboré à ce magazine voulaient innover dans le domaine de la science-fiction et briser ainsi certaines conventions narratives instituées et respectées massivement par les auteurs américains de pulp fiction. Méfiants à l'égard de l'automation, ils s'interrogeaient sur les liens de plus en plus étroits entre l'être humain et la technologie. Pour eux, les machines qui envahissent l'environnement déshumanisent l'être, transforment ses rapports interpersonnels et lui enlèvent tout contrôle sur sa vie et sur le monde ${ }^{10}$. Bien que L'̂̀le de béton se distingue de la tradition expérimentale des publications de New Worlds puisqu'il ne s'agit pas à proprement parler d'un récit de sciencefiction, il participe d'une même problématique. Il procède effectivement d'une critique de la technicisation du monde, principalement des transports. Depuis quelques décennies, l'accident de la route s'avère une importante cause de décès dans les pays industrialisés. En 1972, par exemple, un an avant la première publication de Lîle de béton, "la route a fait sur la planète 250000 tués et 7500000 blessés " ${ }^{11}$. Avec les deux accidents de Maitland et celui de Proctor, le roman rend compte des dangers associés aux transports routiers. Ce n'est toutefois pas l'automobile en tant que telle qui menace la vie, mais son omniprésence ainsi qu'une conduite téméraire. Puisque l'univers statique de l'île engendre la mort, le maintien d'une certaine vitesse paraît indispensable à la survie. Mais cette vitesse transforme l'essence de l'être, l'invite à faire des excès, à rechercher des expériences exaltantes: "Il se rendait compte qu'une moitié de son cerveau se régalait de cette ivresse ou de ce délire, et durement, il parvint à maîtriser cette agitation de maniaque" (p. 21). Le héros est partagé entre un désir de braver le danger pour se sentir vivant tout en frôlant la mort et une prudence qui le garde en vie mais le paralyse. Cette schizophrénie contamine le texte de multiples façons. Entre la vitesse et l'inertie, le corps et la machine, l'intériorité et l'extériorité, la vie et la mort, ce roman de trajets, court mais répétitif, faisant alterner des descriptions précises et un style elliptique, ne cesse de mettre en rapport des oppositions. En outre, comme je l'ai déjà indiqué,

10 Colin Greenland, The Entropy Exbibition: Michael Moorcock and the British "New Wave * in Science Fiction, Londres, Routledge \& Kegan Paul, 1983, p. 168.

11 Jean-François Lemaire, Les accidents de la route, Paris, Presses universitaires de France, coll. "Que sais-je?", 1975, p. 4. 
16

l'univers de l'île peut aussi bien représenter le passé que le futur. Près de vingt-cinq ans après la publication du roman, on y retrouve en effet différents éléments concourant à une anticipation de la réalité technologique actuelle.

Les nouveaux réseaux de communication permettent aux individus de vivre en société tout en s'isolant, de voyager et de s'informer tout en restant immobiles. Conséquemment, la matérialité du corps et la mémoire deviennent de plus en plus accessoires. Paul Virilio prévoit que cette possibilité de se réunir à distance engendrera une désurbanisation de l'espace, une désertification des villes:

Réalisation d'une inertie domiciliaire, la généralisation des techniques du contrôle d'environnement aboutirait à l'isolement comportemental, au renforcement d'une insularité dont la ville a toujours été menacée, la distinction entre l'"îlot " et le "ghetto" demeurant précaire. ${ }^{12}$

Bien qu'on retrouve peu de références dans le roman aux technologies de la communication, la situation de Maitland n'est pas sans préfigurer les transformations socioculturelles et environnementales que Virilio annonce. On a en effet l'impression que l'île "avec son cinéma, son imprimerie, son abri anti-aérien, ses maisons et ses rues en ruine - est une sorte de ville désertée dans laquelle se forme un ghetto d'êtres incapables de s'adapter à la réalité technologique ou, comme dans le cas de Jane, à composer avec ses exigences. Dans un monde où la matérialité des villes, l'extériorité spatiale, cèdera bientôt le pas à "l'immatérialité de ces "télécontinents" constitués par les flux incessants de données" ${ }^{13}$, Maitland, architecte, n'est sans doute plus à sa place et se fait mettre à l'écart. Les autoroutes qui encerclent l'île apparaissent comme autant de réseaux électroniques favorisant la circulation de l'information et au milieu desquels Maitland est piégé. Dans cette perspective, il n'est pas surprenant que le corps soit démembré, que sa matérialité s'estompe: "Son corps, il se mit à le décortiquer, pour s'en débarrasser: supprimée la cuisse blessée, supprimées les deux jambes, effacées la poitrine, les côtes fêlées, gommé le diaphragme cuisant. (p. 70). Et si Maitland cherche à oublier son passé, à se débarrasser de ses souvenirs, si "son cerveau n'absorb[e] presque plus rien" (p. 173), c'est peut-être en

12 Paul Virilio, La vitesse de libération, Paris, Galilée, 1995, p. 34.

13 Ibid., p. 101. 
partie parce que l'essor des machines à communiquer et à penser rend presque inutile tout effort de mémorisation. L'accident projette le héros dans un univers parallèle qui s'avère peut-être davantage virtuel que réel: "Un pneu usé, un coup sur le crâne, et on sort du monde réel" (p. 24). De ce point de vue, la vitesse recherchée dépasse celle permise par l'accélération des transports, elle rejoint celle de la lumière, ne s'apprécie plus en kilomètres/heure mais en milliers de kilomètres par seconde. Après que sa voiture se soit immobilisée, Maitland parcourt un autre trajet, cette fois-ci à l'intérieur de son crâne, l'exploration de cette île qui "se mod[èle] sur son cerveau" (p. 70) servant de prétexte à la réflexion et à la réminiscence. Or, "Du moteur au pare-brise, du pare-brise à l'ordinateur, la voiture du futur fonce vers la vitesse la plus absolue: celle de la pensée "1/4. En ce sens, Maitland atteint peut-être au cours de son voyage introspectif une vitesse limite frôlant l'immédiateté.

Avec l'invention des micro-ordinateurs personnels et la prolifération des réseaux informatiques depuis une quinzaine d'années, l'importance accordée à la vitesse et à la technologie dans L'île de béton ne peut plus être pensée sans que se profilent de nouveaux réseaux de sens. Dans cet univers scindé, oscillant entre le passé et le futur, la schizophrénie du héros, comme celle qui traverse le texte - lui-même à la frontière du roman actuel et de la science-fiction - évoque l'ubiquité de l'être désincarné par l'accélération des communications. Non pas inerte mais soumise à une entropie croissante, annonçant une déperdition énergétique accompagnée d'une désintégration sociale, l'île s'inscrit comme une représentation apocalyptique d'un futur imminent, une critique de la technicisation du monde où la vitesse, limite, altère l'esprit et réincarne l'être dans la machine. Malgré le statisme apparent de l'île, on voit comment la vitesse - d'abord celle des transports routiers puis celle de la lumière - façonne l'univers de Maitland, influe sur ses perceptions, sa conception du corps, de l'environnement et des rapports sociaux, transforme en fait sa vision du monde comme elle ne cesse de métamorphoser celle de tout un chacun depuis environ un siècle et demi.

14 Christophe Tison, L'ère du vite, Paris, Balland, 1989, p. 11. 